\title{
Reactions to threatening health messages
}

\author{
Gill A ten Hoor ${ }^{1 *}$, Gjalt-Jorn Y Peters ${ }^{2}$, Janice Kalagi ${ }^{1}$, Lianne de Groot ${ }^{1}$, Karlijne Grootjans ${ }^{1}$, Alexander Huschens ${ }^{1}$, \\ Constanze Köhninger ${ }^{1}$, Lizan Kölgen ${ }^{1}$, Isabelle Pelssers ${ }^{1}$, Toby Schütt' ${ }^{1}$, Sophia Thomas ${ }^{1}$, Robert AC Ruiter ${ }^{1}$ \\ and Gerjo Kok ${ }^{1}$
}

\begin{abstract}
Background: Threatening health messages that focus on severity are popular, but frequently have no effect or even a counterproductive effect on behavior change. This paradox (i.e. wide application despite low effectiveness) may be partly explained by the intuitive appeal of threatening communication: it may be hard to predict the defensive reactions occurring in response to fear appeals. We examine this hypothesis by using two studies by Brown and colleagues, which provide evidence that threatening health messages in the form of distressing imagery in anti-smoking and anti-alcohol campaigns cause defensive reactions.
\end{abstract}

Methods: We simulated both Brown et al. experiments, asking participants to estimate the reactions of the original study subjects to the threatening health information $(n=93)$. Afterwards, we presented the actual original study outcomes. One week later, we assessed whether this knowledge of the actual study outcomes helped participants to more successfully estimate the effectiveness of the threatening health information $(n=72)$.

Results: Results showed that participants were initially convinced of the effectiveness of threatening health messages and were unable to anticipate the defensive reactions that in fact occurred. Furthermore, these estimates did not improve after participants had been explained the dynamics of threatening communication as well as what the effects of the threatening communication had been in reality.

Conclusions: These findings are consistent with the hypothesis that the effectiveness of threatening health messages is intuitively appealing. What is more, providing empirical evidence against the use of threatening health messages has very little effect on this intuitive appeal.

Keywords: Threatening health messages, Defensive reactions, Smokers, Drinkers

\section{Background}

Threatening health messages that focus on severity in an emotional way are often used as a strategy to reduce unhealthy behaviors $[1,2]$. However, people rarely change their behavior as a result of threatening health messages [3]. Generally, threatening health messages frequently result in defensive reactions [4-6], (Van 't Riet J, Ruiter RAC, Defensive reactions to health-promoting information: An overview and implications for future research. Submitted.) which cause them to have no effect [3,7], or, on occasion, to have a counterproductive effect [3]. Brain imaging techniques have confirmed that people who are most at risk pay the least attention to threatening messages $[8,9]$.

\footnotetext{
* Correspondence: Gill.tenHoor@maastrichtuniversity.nl

${ }^{1}$ Dept. of Work \& Social Psychology, Faculty of Psychology \& Neuroscience, Maastricht University, P.O. Box 616, 6200, MD Maastricht, The Netherlands Full list of author information is available at the end of the article
}

Reviews of the limited effectiveness of threatening health messages have been available for over ten years [3,10-15], obviously without much impact on the attractiveness of using it in behavior change interventions. The most recent meta-analysis resolved a number of problems of previous meta-analyses, finding clear evidence for a significant interaction between threat and efficacy such that threat only had an effect under high efficacy and efficacy only had an effect under high threat [3]. This meta-analysis also showed that under low efficacy the effect of threat was negative and almost significant, which means that if an intervention developer is not very certain that either the target population is high in response and self-efficacy, or that a given intervention will manage to considerably increase both response and self-efficacy, threatening messages should be avoided.

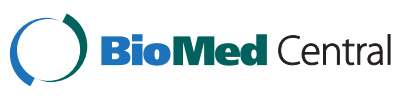


Brown and colleagues have shown that distressing messages that emphasize the severity of a health threat cause defensive reactions. In two studies, the subjects (smokers [16] and regular alcohol consumers [17]) were presented with booklets containing either highly distressing pictures or less distressing pictures. After reading these booklets, the participants rated their own risk and their perceived effectiveness of the booklets. Results of the smoking study [16] showed that rather than having increased their risk perceptions, participants who read distressing booklets reported lowered risk perceptions and evaluated the messages more negatively than participants in the less distressing condition. Results of the alcohol study [17] showed similar effects, although here the defensive reactions were only significant in those regular drinkers who reported both a greater dispositional denial (as measured by the COPE denial scale [18]) and more alcohol-related problems (as measured by a five-item version of the Alcohol Use Disorders Identification Test, AUDIT [19]).

Despite the fact that threatening health messages lead to defensive reactions among those most in need of change, the use of these messages remains well-accepted, even among health professionals [1,2]. So far, limited empirical evidence is available as to why severity-based threatening health messages remain popular despite the distribution of information regarding their ineffectiveness, and as to what measures can be taken to reduce this popularity. In this study, first, we assessed participants' knowledge of the effectiveness of threatening health messages, in particular whether or not they would expect threatening health information to cause defensive reactions. Second, we examined whether presenting participants with information about the actual effects that were found in the Brown et al. studies would change their estimates of the effectiveness of threatening health messages in behavior change programs. To study these research questions, we first copied the materials from the two studies by Brown and colleagues to assess whether participants (students, as in the Brown et al. studies) were able to predict the defensive reactions found in these studies. Then, regardless of their answers, we informed the students about the actual outcomes of the two simulated studies. One week later, participants were again asked to estimate the effectiveness of a threatening message, albeit for a message about a different behavior. Based on the popularity of threatening health messages, we hypothesized that participants expect threatening health information to be effective and that they are unable to predict defensive reactions. In addition, we hypothesized that an intervention that provides empirical evidence against the use of threatening health information can curb the perceived effectiveness of threatening health messages.

\section{Methods}

\section{Full disclosure}

Following recent pleas for full disclosure of research materials, data, analyses and output [20,21], the questionnaires (in LimeSurvey XML survey format, .lss), stimulus materials (in Portable Document Format, .pdf), and the intervention (in the LimeSurvey files) are combined in a .zip archive as Additional file 1 and in a scientific repository at http://sciencerep.org/3/.

\section{Participants}

In total, 134 university students (18 to 29 years of age) enrolled in different curricula at different universities were approached through personal contact with the request to participate in an experiment about threatening health messages. Psychology students were excluded, as it was likely they had already been exposed to the information we would provide in the study. Of the 134 invited participants, 93 participants completed the first part of the study $\left(t_{0}\right.$; see Figure 1$)$, with a mean age of, 21.63 years (median 21, standard deviation 2.1), of whom 52 were female. Of these, 44 participants read the alcohol booklets (see the Procedure section) and 49 participants read the smoking booklets. Most participants ( $n=71$ ) completed the second part of the study $\left(t_{1}\right)$, where 32 rated the alcohol booklets and 38 rated the alcohol booklets (see Procedure section). Among the 71 participants who completed both parts of the study, three $€ 50$ gift vouchers were raffled. The experimental materials and procedure were approved by the Ethics Committee of the Faculty of Psychology and Neuroscience, Maastricht University.

\section{Procedure}

Upon agreeing to participate, participants were asked for their e-mail address after which they received a personalized link to the first part of the online experiment. The

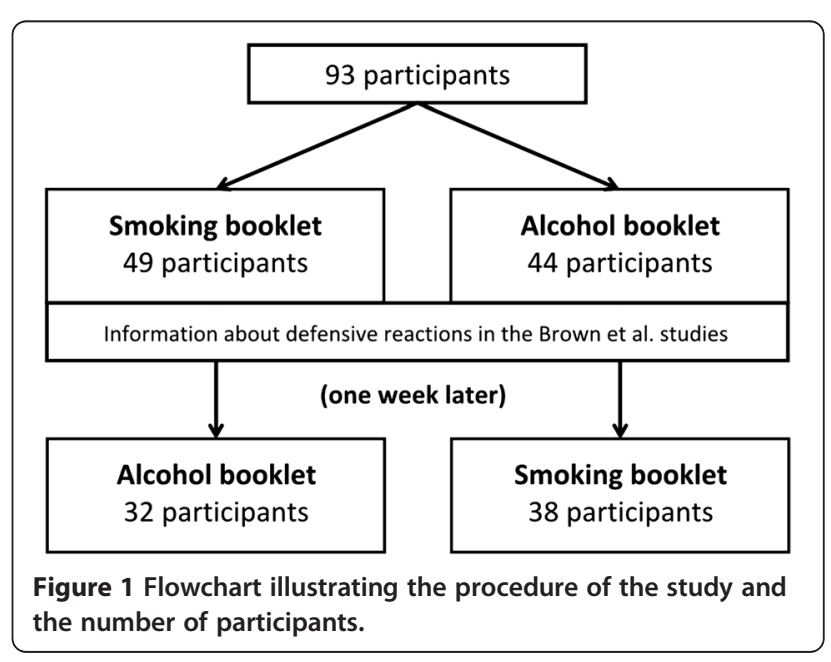


experiment was presented using the open source package LimeSurvey. The link opened an instance of LimeSurvey [22] where participants were asked to participate in two tests $\left(t_{0}\right.$ and $\left.t_{1}\right)$ with an intervention in between (see Figure 1). Subsequently, participants gave their informed consent by clicking on the appropriate button and were randomized into one of two groups. Participants in the first group were presented with the two booklets about smoking, whereas participants in the other group were presented with the two booklets about drinking. In both groups, the participants read both one booklet with highly threatening images (HT) and one with less threatening images (LT). At the start of $t_{0}$, the participants were first informed about the procedures of the original experiment (either the smoking or the drinking study, according to a participant's group), but they were given no information about the results. Then, participants were informed about the characteristics of the subjects of the original experiments (either people who smoke at least one cigarette per day or people who drink at least six glasses of alcohol at least twice a week) and instructed to estimate how the original subjects rated the booklets. During the entire test, participants were able to switch between the LT and HT booklets. After the participants read the booklets, their gender and age were measured, followed by questions assessing their predictions as to how the subjects in the original experiments [16,17] evaluated the health messages and how they responded to questions about personal risk and emotional impact. Finally, participants' own smoking and drinking behaviors were measured.

Immediately after $t_{0}$, participants were presented with an intervention consisting of information about the actual defensive reactions to threatening health messages in the Brown et al. study and an explanation of the dynamics of processing threatening information (see Intervention section). One week after $t_{0}, t_{1}$ followed. Participants were asked not to talk about the study in between $t_{0}$ and $t_{1}$. At $t_{1}$, participants who read the booklets about smoking during $t_{0}$ now read the booklets about alcohol and vice versa, to make sure that we measured understanding of the dynamics of threatening messages on a general level, instead of simple recognition. After participants inspected the booklets, the same measures were completed as at $t_{0}$. At the end of the study, participants were debriefed.

\section{Stimulus materials}

To maximize accuracy of our simulation, the materials (booklets and questionnaires) from the original experiments [16,17] were copied as accurately as possible. However, there were two differences in the procedure: 1) our participants were asked to rate both booklets (high threat messages and low threat messages) instead of a single booklet (see Measures section) and 2) our study was online. Furthermore, our participants used 7-point Likert scales ( -3 to 3 ) for all questions, where in the Brown et al. studies, a variety of scales was used. With permission from Brown, two pairs of booklets were copied from the original studies $[16,17]$. The booklets provided the same information about the negative consequences of smoking or drinking, but contained different images. In the HT booklets, the pictures were highly distressing. In the smoking booklet, pictures presented a smokers lung; surgically removed cancer of the lower jaw; a gangrenous foot; and lung emphysema. In the alcohol booklet, pictures were presented of an enlarged liver; mouth and throat cancer; chronic pancreatitis; and a fatal car accident. In the LT booklets, less distressing pictures were shown. The smoking booklet contained pictures of cigarettes with the text "smoking causes cancer in a lot of places (including the butt)"; cigarettes in a beer glass with the text "avoid drinking with killers"; a cigarette in the shape of an injection needle with the text "cigarettes kill 17,500 more Australians each year than heroin"; and a picture of cigarettes in the shape of a hand with the middle finger up "The signal from tobacco companies is loud and clear". The alcohol booklet contained a picture of a fallen glass ('spilling beer'); a picture of a man drinking beer ('overuse of alcohol has a range of health effects'); a picture of a toast with beer ('drinking in rounds can increase consumption'); pictures of cars ('drink driving can have fatal consequences'), and an advertisement against drink driving. Participants could switch between the original English booklets and Dutch translations.

\section{Measures}

The measures were replicated from the original studies $[16,17]$. Each participant provided ratings for both the HT and LT booklets (see Figure 2). Participants were told that it was not important whether they smoked cigarettes or drank alcohol or not, and they were explicitly asked to identify themselves with the smoking or drinking subjects in the original experiments, rather than answering the questions for themselves. As a manipulation check, participants were asked to rate the estimated emotional impact of the booklets. Scores on items that measured the same construct were averaged into one scale. The internal consistency of these scales was good ( $\omega_{\text {total }}$ ranged from .81 to .98; the Greatest Lower Bound, GLB, ranged from .84 to .99; we computed these measures rather than Cronbach's $\alpha$ because of the severe problems of the latter, see e.g. $[23,24])$. The interested reader can examine the Cronbach's alpha's in our output file in Additional file 1 or at http:// sciencerep.org/3/. All questions were formulated as depicted in Figure 2. 


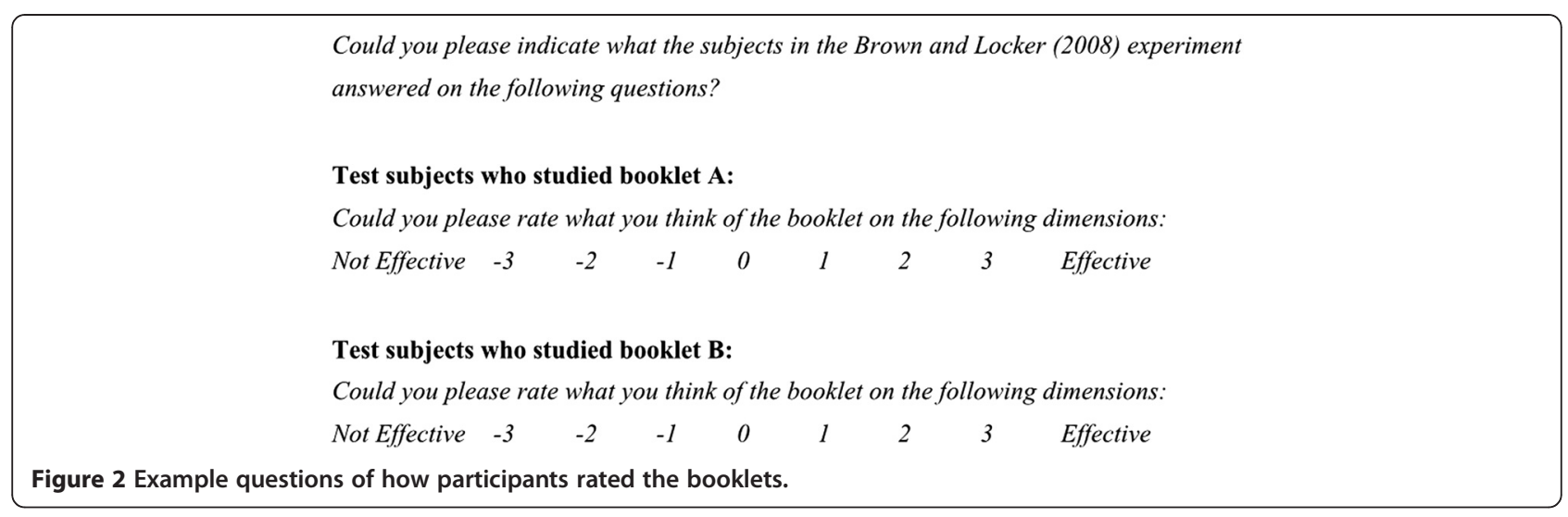

\section{Emotional impact}

Participants estimated the emotional impact that the materials had on the participants in the original studies by answering three items that asked to what extent participants in the original study reported feeling distressed, anxious and uneasy. These items used 7-point Likert scales ranging from -3 (extremely) to 3 (not at all).

\section{Evaluation of the booklets}

Eight 7-point semantic differentials (ranging from -3 to 3 ) measured the extent to which participants thought that the original subjects rated the booklets on the following dimensions: persuasive-not persuasive, bad-good, clever-stupid, not effective-effective, absolutely makes me want to quit smoking/decrease drinking-absolutely does not make me want to quit smoking/decrease drinking, no weaknessessignificant weaknesses, no strengths-significant strengths, very important message-unimportant message. Item scores were recoded and averaged such that higher scores on the aggregate scale reflected higher estimated booklet effectiveness.

\section{Risk perception}

Our participants estimated original study participants' perceived likelihoods of getting a tobacco or alcohol related disease or problem using six items for smoking and eight items for alcohol consumption. The tobacco related diseases or problems (heart attack, bronchitis, lunch cancer, stroke, cancer of the throat or mouth, emphysema) and alcohol related diseases or problems (serious difficulties in an intimate relationship, become an addict, serious liver disease, the degree to which alcohol makes one insult or be rude to people, trouble with family relationships, serious injuries, conflicts or difficulties with friends, and withdrawal symptoms) were all rated on a 7-point Likert scale, ranging from -3 (no chance) to 3 (completely certain), where higher scores reflected a higher estimated risk perception.

Finally, participants were asked about their own smoking and drinking behavior. Smoking behavior was assessed by questions about the smoking status (yes/no), how many cigarettes are smoked during the week and weekend, and the number of previous quit attempts. Alcohol drinking behavior was measured using the AUDIT [19].

\section{Intervention}

The intervention was designed to teach people about the potential negative effects of threatening health messages by providing understandable scientific evidence and explaining why threatening messages are ineffective. The information included a brief explanation of the experimental procedure and outcomes of the Brown et al. studies, showing that fear arousing messages lead to defensive reactions that render them ineffective. In addition, the intervention explained how threatening information is processed. As we estimated that the prevalent model dealing with threatening communication (the Extended Parallel Process Model; [25]) would be conceptually too complex for our participants, we explained the dynamics of threatening communication using cognitive dissonance reduction. Specifically, we explained that people experience cognitive dissonance in situations in which they feel a disharmony between their behavior and beliefs, and that to reduce this disharmony, defensive mechanisms (such as changing beliefs regarding susceptibility to a risk) are used when one's selfefficacy regarding changing the relevant behavior is low [7]. The message suggested that it would be more effective to motivate people to reduce risk behavior by telling them how they can change their unhealthy behavior, instead of scaring them.

\section{Data analysis}

Analyses were conducted using R [26]. In acknowledgement of recent concerns regarding lack of disclosure in scientific research [27], and to aid future meta-analyses, all data, R scripts, and output files are available at http:// sciencerep.org/3/ and in the Additional file 1 archive. We report $80 \%$ confidence intervals of effect sizes (following Cohen's recommendation; [28]) rather than 
Null Hypothesis Significance Testing (NHST) outcomes, based on the problems of NHST (e.g. [29]) and pleas to remedy this problematic approach (e.g. [30,31]). However, interested readers can always view the NHST statistics ( $t$ values, degrees of freedom, and p-values) in the output file that is available as a text-file at http://sciencerep.org/3/ and in Additional file 1. Because not all readers may be used to working with confidence intervals yet, we will also report whether associations are significant or not; corresponding values for $t$ and the degrees of freedom can be found in the text-file with the original output at http:// sciencerep.org/3/ and in Additional file 1.

We first conducted manipulation checks, comparing the estimated emotional impact for the LT and HT booklets. Then, difference scores for the LT and HT ratings for the evaluation and risk perceptions estimates were computed, and potential covariates were explored by examining the bivariate associations of age, sex and behavior (smoking and alcohol use) with the difference scores of evaluation and risk perception estimates (no covariates were associated to these difference scores; see the output file). To test the first hypothesis (LT booklets are rated lower than HT booklets), we computed confidence intervals for Cohen's $d$ for the differences in ratings between the LT and the HT booklets at $t_{0}$. Then, to test the second hypothesis, we computed confidence intervals for Cohen's $d$ for the change in mean HT-LT rating differences between $t_{0}$ and $t_{1}$. In these analyses, we used the pooled standard deviation if variances were equal, and the standard deviation from the largest of the two samples if they were not (see our analysis script at http://sciencerep.org/3/ or in Additional file 1).

\section{Results}

Participants' ratings of the LT and HT booklets at $t_{0}$ and $t_{1}$, and the differences between the LT and HT ratings, are shown in Table 1 and Figure 3.

\section{Manipulation checks}

Participants accurately predicted that participants experienced the HT booklets as more distressing than the LT booklets, with estimated emotional distress differences of around one standard deviation at $t_{0}$ for both behaviors and at $t_{1}$ for alcohol (Cohen's $d$ confidence intervals ranging from 0.86 to 1.76), but a difference of only a fifth to a ninetieth standard deviation for alcohol at $t_{1}$ (Cohen's $d$ 0.17-0.93). Null Hypothesis Significance Testing (NHTS) revealed $p$-values of .002 or smaller.

\section{Differences in estimated ratings $\left(t_{0}\right)$ \\ Estimated evaluation}

Our participants estimated that the participants in the original studies rated the HT booklet higher. For alcohol, the HT booklet was rated especially high, around one standard deviation higher (Cohen's $d$ 0.72-1.30); and for smoking around half a standard deviation (Cohen's d 0.15-0.67). NHST revealed respective $p$-values of $<.001$ and .044 .

\section{Estimated risk perception}

Again, our participants estimated that the participants in the original studies rated the HT booklet higher. The HT booklet was rated around half a standard deviation higher for both alcohol (Cohen's $d$ 0.31-0.87) and smoking (Cohen's d 0.21-0.73). NHST revealed respective $p$-values of .007 and .022 .

Table 1 Means and standard deviations of estimated emotional impact, estimated evaluation, and estimated risk perception for the LT and HT booklets in the current study, and the means and standard deviations found in the original studies of Brown et al

\begin{tabular}{|c|c|c|c|c|c|c|}
\hline & & & $\begin{array}{l}\text { LT booklet } \\
\text { mean }(\mathrm{sd})\end{array}$ & $\begin{array}{l}\text { HT booklet } \\
\text { mean }(\mathrm{sd})\end{array}$ & $\begin{array}{l}\text { LT booklet } \\
\text { B\&S/B\&L mean (sd) }\end{array}$ & $\begin{array}{l}\text { HT booklet } \\
\text { B\&S/B\&L mean (sd) }\end{array}$ \\
\hline \multirow[t]{6}{*}{$t_{0}$} & Alcohol & Emotional impact & $-1.18(1.33)$ & $1.20(1.32)$ & & \\
\hline & $(n=44)$ & Evaluation & $-0.33(1.12)$ & 0.79 (1.07) & $0.26(0.67)$ & $0.53(0.38)$ \\
\hline & & Risk perception & $-0.57(1.07)$ & $0.12(1.25)$ & $-2.04(0.71)$ & $-1.8(0.83)$ \\
\hline & Smoking & Emotional impact & $-0.79(1.27)$ & $1.10(1.48)$ & & \\
\hline & $(n=49)$ & Evaluation & $0.30(0.85)$ & $0.65(0.86)$ & $0.44(1.17)$ & $1.15(0.99)$ \\
\hline & & Risk perception & $0.32(1.18)$ & $0.86(1.11)$ & $-0.52(1.34)$ & $0.22(1.45)$ \\
\hline \multirow[t]{6}{*}{$t_{1}$} & Alcohol & Emotional impact & $-0.91(1.22)$ & $0.81(1.28)$ & & \\
\hline & $(n=32)$ & Evaluation & $0.06(0.91)$ & $0.52(1.13)$ & $0.26(0.67)$ & $0.53(0.38)$ \\
\hline & & Risk perception & $-0.41(1.13)$ & $0.03(1.29)$ & $-2.04(0.71)$ & $-1.8(0.83)$ \\
\hline & Smoking & Emotional impact & $-0.25(1.24)$ & $1.03(1.49)$ & & \\
\hline & $(n=38)$ & Evaluation & $0.34(0.91)$ & $0.59(1.17)$ & $0.44(1.17)$ & $1.15(0.99)$ \\
\hline & & Risk perception & $0.27(1.26)$ & $0.45(1.44)$ & $0.52(1.34)$ & $0.32(1.45)$ \\
\hline
\end{tabular}

Note: participants who evaluated the alcohol booklet at $t_{0}$, evaluated the smoking booklet at $t_{1}$ and vice versa. To enable comparison, scores from the Brown et al. studies were recoded to the metric used in the current study (i.e. ranging from -3 to 3 ). 


\section{Intervention effects}

To test the effects of our intervention, we first subtracted participants' estimated ratings of the LT booklet of the estimated ratings of the HT booklets (i.e. yielding the difference score, the deviation of which from zero we tested in the previous paragraph). We then compared these difference scores at $t_{0}$ (before our intervention) and $t_{1}$ (after our intervention). Note that this was a comparison of two independent groups, because the participants who examined the alcohol booklets at $t_{0}$, examined the smoking booklets at $t_{1}$, and vice versa. The Cohen's $d$ s for this comparison are positive when participants' mean ratings increased between $t_{0}$ and $t_{1}$, and negative when they decreased (our hypothesis is that the ratings will decrease).

Although participants did appear to decrease their ratings, these decreases were very minimal. For estimated effectiveness, Cohen's d confidence intervals ranged from -.74 to -.14 for alcohol and from -.35 to 0.21 for smoking; and for estimated risk perception, from -0.53 to 0.07 for alcohol and from -0.61 to -0.05 for smoking. None of these differences was significant according to NHST procedures, with $p$-values of .061, $.739, .327$ and .290 , respectively.

\section{Comparisons with the Brown et al. Studies}

Participants in the Brown et al. studies evaluated the HT booklets lower than the LT booklets, and the HT booklets had a negative effect on their risk perception. Our participants' estimations were exactly the opposite: they estimated that the HT booklets would be evaluated more positively, and that the HT booklets would cause more increases in risk perception (see Figure 3).

\section{Discussion}

Regardless of the fact that there is much evidence about the ineffectiveness of threatening health messages, they remain widely applied in behavior change campaigns. Our study explored a possible reason: people are unable to predict the effects of threatening health messages. Most people seem convinced that threatening health messages work, and not able or willing to change this belief.

We have shown that people are unable to predict the defensive reactions to threatening health messages that occurred in the Brown and Smith and Brown and Locker studies [16,17]. Our participants predicted the emotional reactions to the booklets correctly (i.e. highly threatening messages cause higher anxiety). However, our participants did not predict the effect on estimated perceived risks and evaluations of the booklets correctly. Those were estimated to be higher after exposure to fear arousing messages, which directly contrasts with the outcomes of the Brown and Smith [16] and Brown and Locker [17] studies, which found either no effects or adverse effects of threatening health messages.

In contrast to our last hypothesis, the beliefs about the effectiveness of threatening health messages barely changed as a result of a confrontation with the empirical evidence and an explanation of the relevant dynamics. This implies that people do not readily internalize scientific evidence regarding fear appeal effectiveness, remaining convinced that threatening health messages lead to an increased risk perception. This reluctance of professionals to change when presented with evidence inconsistent with their beliefs is in line with previous results [32,33]; successfully changing professionals beliefs seems to require interventions that are a lot more sophisticated than our brief text [34].

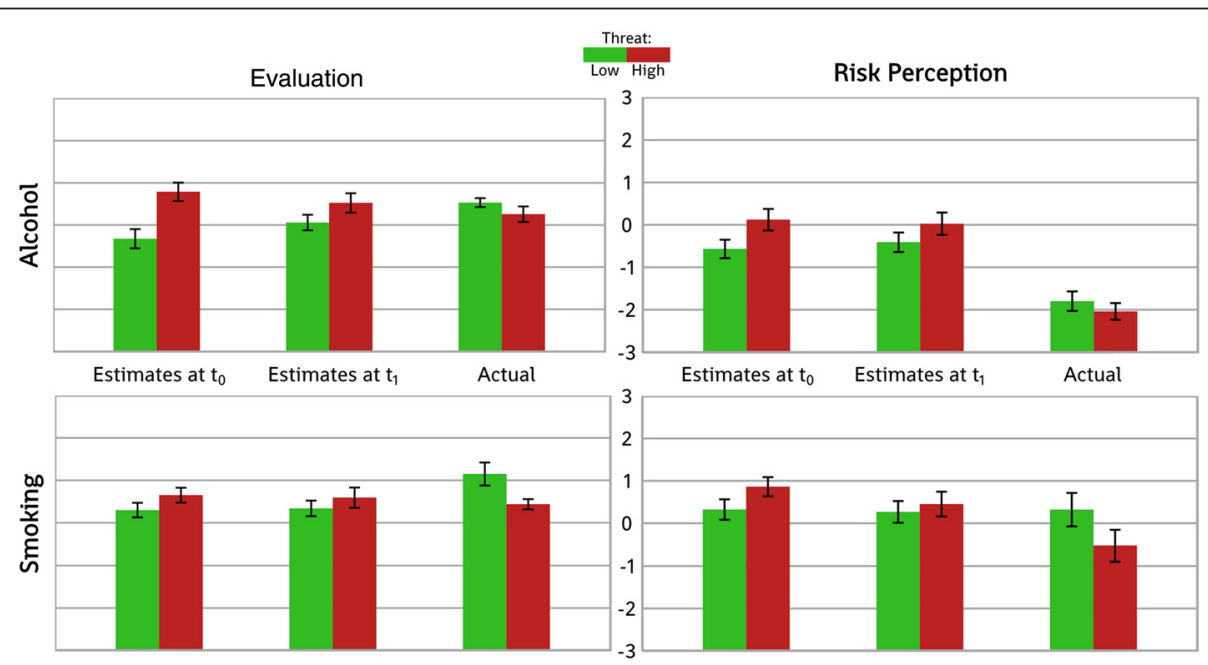

Figure 3 The mean ratings for estimated risk perception and evaluation, compared to the actual outcomes in the Brown et al. studies. The error bars indicate $95 \%$ confidence intervals around the means. 
There is one alternative explanation of this result. Perhaps participants do understand how threatening communication is processed; perhaps their error is not their assumption that threatening communications are generally effective, but their assumption that in general, efficacy levels of smokers and alcohol abusers are high. In this case, the results would be the same; participants would estimate threatening communication to be effective, while being aware that this is conditional upon high efficacy. This would still represent an estimation error, as efficacy is generally low in those exhibiting risk behaviors (see for example [35]). Although this alternative explanation would explain why our intervention did not have an effect, it is inconsistent with both anecdotal evidence from our study and evidence from another study. Some participants reported in a debriefing conversation that they simply did not believe that fear arousing messages are ineffective. They stated that intuitively, it felt right that threatening health messages influence behaviour and even clear and explicit scientific evidence could not convince them otherwise. This is in line with results found in Peters, Ruiter, \& Kok [36], who found that public health professionals, advertising professionals, and decision makers often favour threatening health messages, assuming erroneously that those messages attract attention and prompt self reflection through confrontation, which ultimately leads to behaviour change.

This study suffered from a number of limitations. First, we did not check whether people actually read the provided information regarding the ineffectiveness of threatening health messages carefully enough. Note, however, that this reflects real life, where interventions aiming to educate key stakeholders as to fear appeal ineffectiveness are also not guaranteed of recipients' undivided attention. To gauge how seriously participants read the health information, we asked random participants about their experience and opinion about the given information during the debriefing, and indeed, some reported that they did not put much effort in reading the information (others indicated that they did, and some volunteered that they changed their beliefs). Another potential limitation is the fact that our sample consisted entirely of university students. This problem, the 'sophomore bias', has existed since around 1960 [37], and is by no means exclusive to the current investigation. Although the use of students as participants has recently been defended as well [38], we would like to raise an observation of Sears (1986, p. 527), namely that "the human being of strong and irrational passions [...] is not that of contemporary social psychology". This reasoning would imply that if anything, using university students has worked against us: as students suffer less from "irrational passions", one would have expected these rational participants to have embraced the evidence we provided in our intervention, at least more so than a different sample.

Another aspect of the current study that strengthens its conclusions, is that in the current study, participants rated both booklets simultaneously; and the LT booklets can be considered persuasive in their own right. Thus, we can exclude the potential interpretation that the higher ratings of the HT booklets reflect the fact that participants expect threatening communication to work better than nothing; rather, the higher ratings reflect that participants expect threatening communication to have a stronger effect than another plausibly effective communication.

\section{Conclusion}

In conclusion, people expect threatening health messages to be effective, and this belief is hard to influence by presentation of evidence to the contrary. Clearly, trying to change beliefs about the effectiveness of threatening health messages is not as easy as one might think. As shown in this study, written information with scientific evidence and theory, testifying to the ineffectiveness of threatening health messages, did not affect people's evaluation of either the expected evaluation of threatening health messages, or the expected effect on risk perceptions. Peters et al. [36] proposed that part of the problem may be a lack of adequate alternative behaviour change methods. They suggest that when communicating with professionals, it seems fruitful to provide them with a toolbox of evidence-based behaviour change methods that promote adaptive, rather than defensive, behaviour [39-42]. The results of the current study suggest that in any case, providing scientific theory and empirical evidence does not seem to suffice to diminish the intuitive preference for threatening communications.

\section{Additional file}

Additional file 1: Full disclosure of research materials, data, analyses and output.

\section{Competing interests}

The authors declare that they have no competing interests.

\section{Authors' contributions}

GH, JK, LG, KG, AH, CK, LK, IP, TS, ST and GK conceived the study, and collected and analyzed the data. GH, RR and GK designed the study. GJP analyzed the data. GH, GK, GJP and RR contributed to the interpretation of the data. GH, GJP and GK drafted the manuscript. All authors provided feedback on the manuscript and read and approved the final text.

\section{Acknowledgement}

We are grateful to Stephen Brown, who was willing to donate the materials and measurement instruments from his studies.

\section{Author details}

${ }^{1}$ Dept. of Work \& Social Psychology, Faculty of Psychology \& Neuroscience, Maastricht University, P.O. Box 616, 6200, MD Maastricht, The Netherlands. 
${ }^{2}$ Dept. of Research Methods \& Statistics, Faculty of Psychology, Open University, P.O. Box 2960, 6401, DL Heerlen, The Netherlands.

Received: 20 June 2012 Accepted: 13 November 2012 Published: 21 November 2012

\section{References}

1. Albarracín D, Gillette JC, Earl AN, Glasman LR, Durantini MR, Ho M-H: A test of major assumptions about behaviour change: a comprehensive look at the effects of passive and active hiv-prevention interventions since the beginning of the epidemic. Psycholy Bull 2005, 131:856-897.

2. Cohen EL, Shumate MD, Gold A: Anti-Smoking Media Campaign Messages: Theory and Practice. Health Commun 2007, 22:91-102.

3. Peters G-JY, Ruiter RAC, Kok G: Threatening communication: A critical reanalysis and extension of meta-analyses to determine the behavior change potential of threatening health information. Health Psychol Rev, doi:10.1080/17437199.2012.703527.

4. Croyle RT, Sun YC, Louie DH: Psychological minimization of cholesterol test results: Moderators of appraisal in college students and community residents. Health Psychol 1993, 12:503-507.

5. Good A, Abraham C: Measuring defensive responses to threatening messages: A meta-analysis of measures. Health Psychol Rev 2007, 1:208-229.

6. Liberman A, Chaiken S: Defensive processing of personally relevant health messages. Pers Soc Psychol Bull 1992, 18:669-679.

7. Ruiter RAC, Abraham C, Kok G: Scary warnings and rational precautions: a review of the psychology of fear appeals. Psychol Health 2001, 16:613-630

8. Kessels LTE, Ruiter RAC, Brug J, Jansma BM: The effects of tailored and threatening nutrition information on message attention: Evidence from an event-related potential study. Appetite 2011, 56:32-38.

9. Kessels LTE, Ruiter RAC, Jansma BM: Increased attention but more efficient disengagement: neuroscientific evidence for defensive processing of threatening health information. Health Psychol 2010, 29:346-354.

10. de Hoog N, Stroebe W, de Wit JBF: The impact of vulnerability to and severity of a health risk on processing and acceptance of fear-arousing communications: a meta-analysis. Rev Gen Psychol 2007, 11:258-285.

11. Earl AN, Albarracín D: Nature, decay, and spiraling of the effects of fearinducing arguments and hiv counseling and testing: a meta-analysis of the short- and long-term outcomes of hiv-prevention interventions. Health Psychol 2007, 26:496-506.

12. Floyd DL, Prentice-Dunn S: A meta-analysis of research on protection motivation theory. J App/ Soc Psychol 2000, 30:407-429.

13. Milne S, Sheeran P. Orbell S: Prediction and intervention in health-related behavior: A meta-analytic review of protection motivation theory. J App Soc Psychol 2000, 30:106-143.

14. Ruiter RAC, Kok G: Response to Hammond et al. showing leads to doing, but doing what? the need for experimental pilot-testing. Eur J Public Health 2006, 16:225.

15. Witte $K$, Allen M: A meta-analysis of fear appeals: implications for effective public health campaigns. Health Educ Behav 2000, 27:591-615.

16. Brown SL, Smith EZ: The inhibitory effect of a distressing anti-smoking message on risk perceptions in smokers. Psychol Health 2007, 22:255-268.

17. Brown SL, Locker E: Defensive responses to an emotive anti-alcohol message. Psychol Health 2009, 24:517-528.

18. Carver CS, Scheier MF, Weintraub JK: Assessing coping strategies: A theoretically based approach. J Pers Soc Psychol 1989, 56:267-283.

19. Miles $H$, Winstock $A$, Strang J: Identifying young people who drink too much: The clinical utility of the five-item alcohol use disorders test (AUDIT). Drug Alcohol Rev 2001, 20:9-18.

20. Simmons JP, Nelson LD, Simonsohn U: False-positive psychology: undisclosed flexibility in data collection and analysis allows presenting anything as significant. Psychol Sci 2011, 22(11):1359-1366.

21. Peters G-JY, Abraham CS, Crutzen R: Full disclosure: doing behavioural science necessitates sharing. Eur Health Psychol 2012, 14(4):77-84.

22. LimeSurvey Project Team LimeSurvey: An Open Source survey tool. Hamburg, Germany; Retrieved from http://limesurvey.org

23. Revelle W, Zinbarg RE: Coefficients Alpha, Beta, Omega, and the glb: Comments on Sijtsma. Psychometrika 2008, 74(1):145-154.

24. Sijtsma K: On the Use, the Misuse, and the Very Limited Usefulness of Cronbach's Alpha. Psychometrika 2009, 74(1):107-120.
25. Witte K: Fear control and danger control: A test of the extended parallel process model. Commun Monogr 1994, 61:113-134.

26. R Development Core Team R: A Language and Environment for Statistical Computing: Retrieved from http://www.r-project.org/.

27. Simmons J, Nelson L, Simonsohn U: False-Positive Psychology: Undisclosed Flexibility in Data Collection and Analysis Allows Presenting Anything as Significant. Psychol Sci 2011, 22:1359-1366.

28. Cohen J: Things I Have Learned (So Far). Am Psychol 1990, 45:1304-1132.

29. Masicampo EJ, Lalande DR: A peculiar prevalence of $p$ values just below .05. Q J Exp Psychol 2012, 65(11):2271-2279.

30. Denis DJ: Alternatives to Null Hypothesis Significance Testing. Theory Sci 2003, 4(1):2.

31. Kirk RE: Promoting Good Statistical Practices: Some Suggestions. Educ Psychol Meas 2001, 61(2):213-218.

32. Keijsers JFEM, Bouter LM, Meertens RM, Kessels AGH, Knipschild PG: The impact of back school research on the beliefs of health care professionals: A randomised survey of general health practitioners and physiotherapists. Physiother Theory Pract 1992, 8:79-83.

33. Rovers MM, van der Wilt GJ, van der Bij S, Straatman Kl, Zielhuis GA: Bayes' theorem: A negative example of RCT on grommets in children with glue ear. Eur J Epidemiol 2004, 20:23-28.

34. Boonacker CWB, Hoes AW, Dikhoff M-J, Schilder AGM, Rovers MM: Interventions in health care professionals to improve treatment in children with upper respiratory tract infections. Int J Pediatr Otorhinolaryngol 2010, 74:1113-1121.

35. Hamilton G, Cross D: Occasional Cigarette Smokers: Cue for Harm Reduction Smoking Education. Addict Res 2000, 8(5):419-437.

36. Peters G-JY, Ruiter RAC, Kok G: Threatening communication: A qualitative study of fear appeal effectiveness beliefs among interventionists, policymakers, politicians, scientists and advertisers. Int J Psychol, in Press.

37. Sears DO, Brewer M, Citrin J, Converse PE, Dorr A, Freedman L, Houston JP: College Sophomores in the Laboratory: Influences of a Narrow Data Base on Social Psychology's View of Human Nature. J Pers Soc Psychol 1986, 51(3):515-530

38. Druckman JN, Kam CD. Students as Experimental Participants: A Defense of the 'Narrow DataBase; 2009. Available at SSRN: http://ssrn.com/ abstract=1498843 or http://dx.doi.org/10.2139/ssrn.1498843.

39. Bartholomew LK, Parcel GS, Kok G, Gottlieb NH, Fernández ME: Planning health promotion programs: an Intervention Mapping approach. 3rd edition. San Francisco, CA: Jossey-Bass; 2011

40. De Bruin M, Viechtbauer W, Schaalma HP, Kok G, Abraham C, Hospers HJ: Standard care impact on effects of highly active antiretroviral therapy adherence interventions: A meta-analysis of randomized controlled trials. Arch Intern Med 2010, 170:240-250.

41. Ruiter RAC, Kok G: Planning to frighten people? Think again! In Writing health communication: An evidence-based guide for professionals. Edited by Abraham C, Kools M. London: Sage; in Press.

42. Van Achterberg T, Huisman-De Waal GGJ, Ketelaar NABM, Oostendorp RA, Jacobs JE, Wollersheim HCH: How to promote healthy behavior in patients? A review of systematic reviews. Health Promot Int 2010, 26(2):148-162.

doi:10.1186/1471-2458-12-1011

Cite this article as: ten Hoor et al:: Reactions to threatening health messages. BMC Public Health 2012 12:1011.

\section{Submit your next manuscript to BioMed Central and take full advantage of:}

- Convenient online submission

- Thorough peer review

- No space constraints or color figure charges

- Immediate publication on acceptance

- Inclusion in PubMed, CAS, Scopus and Google Scholar

- Research which is freely available for redistribution 